\title{
Lipo-oligosaccharide immunotyping of Neisseria meningitidis by a whole-cell ELISA with monoclonal antibodies
}

\author{
R. J. P. M. SCHOLTEN*†, B. KUIPERS $\dagger$, H. A. VALKENBURG*, J. DANKERT $\ddagger, W$. D. ZOLLINGER \\ and J. T. POOLMAN† \\ * Institute for Research in Extramural Medicine, Vrije Universiteit, Amsterdam, $\dagger$ Unit of Bacterial Vaccine \\ Development and Pathogenesis Research, National Institute for Public Health and Environmental Protection, \\ Bilthoven, $\ddagger$ Netherlands Reference Laboratory for Bacterial Meningitis, WHO Collaborating Centre, University of \\ Amsterdam/National Institute for Public Health and Environmental Protection, Amsterdam. The Netherlands and \\ $\S$ Walter Reed Army Institute of Research, Walter Reed Army Medical Center, Washington DC, USA
}

\begin{abstract}
Summary. To assess the applicability of a whole-cell ELISA (WCE) with monoclonal antibodies (MAbs) for lipo-oligosaccharide (LOS) immunotyping of Neisseria meningitidis, 675 meningococcal isolates obtained in 1989 and 1990 in the Netherlands and 57 isolates collected in 1974, of which the immunotype had been determined previously by microprecipitation, were analysed. Despite the lack of specific MAbs for L2 and L4, an algorithm was developed for the assignment of immunotypes on the basis of the reaction patterns of the reference strains and these isolates to a combination of 14 MAbs. The immunotypes found by WCE were in accordance with those obtained by microprecipitation and the results from WCE were reproducible. The distribution of immunotypes among isolates of the various serogroups in the Netherlands in 1989-1990 is presented. Based on the reaction patterns of the isolates, two main categories of related immunotypes could be distinguished among isolates of serogroups B and $\mathrm{C}: \mathrm{L} 2 / \mathrm{L} 4$ and $\mathrm{L} 3 / \mathrm{L} 1 / \mathrm{L} 8$. Some isolates of the latter category were of one immunotype, but many isolates expressed one or two additional immunotypes, either strongly or weakly, indicating that the differences in this category are quantitative rather than qualitative. The results of this study have demonstrated that the WCE method for LOS immunotyping is easily applicable and provides better definition of test strains for invitro bactericidal assays and research into pathogenesis.
\end{abstract}

\section{Introduction}

In 1985 , a scheme was proposed for the characterisation of Neisseria meningitidis isolates. ${ }^{1}$ It consisted of a combination of serogroup, serotype, subtype and lipopolysaccharide (LPS) serotype. The name of the last of these was changed to lipo-oligosaccharide (LOS) immunotype (IT) after it had been demonstrated that the oligosaccharide structure of the LPS of the meningococcal outer membrane determined the LPS serotype. ${ }^{2}$ Serogrouping, serotyping and subtyping have proved useful for studying the epidemiology of meningococcal disease, but LOS immunotyping is not regularly incorporated into these studies. LOS immunotyping has been hindered by the complexity of the methods involved ${ }^{3,4}$ but in recent

Received 29 Dec. 1993; revised manuscript received 24 March 1994; accepted 29 March 1994 years an increasing number of monoclonal antibodies (MAbs) has been developed, facilitating immunotyping. ${ }^{5-7}$ However, there is still no MAb available for every IT. Moreover, the interpretation of immunotyping results is difficult, because meningococci often express several ITs, and the expression of ITs may be influenced by growth conditions. ${ }^{3,4.8-11}$ At present, 11 different LOS ITs are recognised: L1-L11, of which L3, L7 and L9 are closely related immunochemically. ${ }^{3,4,8,12}$ The chemical structure of the terminal structures of L1-L6 and L8 has been elucidated. ${ }^{13-18}$ Recently, two new LOS ITs (L12 and L13) were described among meningococci of serogroup A, but it is not yet clear whether they represent unique LOS structures. L9-L11 are found predominantly among serogroup A meningococci, and L2-L4 among serogroups B and C. ${ }^{3-5,8,19,20}$ Knowledge of the distribution of LOS ITs among serogroup 
B meningococci might become of special interest, because the LOS is a potential vaccine candidate. ${ }^{21}$

The aim of this study was to evaluate the applicability of the method of whole-cell ELISA (WCE) for LOS immunotyping with MAbs. Despite the lack of specific MAbs for every IT, it was possible to develop an algorithm for the assignment of immunotypes, based on the WCE results. The ITs of 57 patient strains collected in 1974, were determined by WCE, and the results were compared with the previously described results of microprecipitation. ${ }^{4}$ The reproducibility of the WCE method was assessed by comparing the immunotyping results of two separate cultures of 116 isolates. The distribution of LOS ITs among 563 consecutive patient strains, obtained in the Netherlands in 1989-1990, and their association with the serogroups, serotypes and subtypes is reported.

\section{Materials and methods}

\section{Bacterial isolates}

Since 1958, isolates of $N$. meningitidis from the blood or cerebrospinal fluid (CSF) of patients in the Netherlands with meningococcal disease have been sent on chocolate agar slants from regional laboratories to the Netherlands Reference Laboratory for Bacterial Meningitis in Amsterdam. Upon arrival, the isolates are serogrouped $\mathrm{d}^{22}$ and stored at $-70^{\circ} \mathrm{C}$ on glass beads as suspensions in glycerol $15 \%$ broth.

In this study, the isolates from 563 consecutive patients with meningococcal disease, collected in the period from 1989 to 1990 , were examined. Isolates from both the blood and CSF were obtained from 112 patients, giving a total of 675 isolates for analysis. Also included were 57 patient isolates, collected in 1974, of which the IT had been determined previously by microprecipitation. ${ }^{4}$ The following reference strains were used: 126E (L1), 35E (L2), 6275 (L3), 89I (L4), M981 (L5), M992 (L6), 6155 (L7), M978 (L8), 120M (L9), 7880 (L10) and 7889 (L11).

The isolates were cultured overnight on chocolate agar plates at $37^{\circ} \mathrm{C}$ in a humid atmosphere containing $\mathrm{CO}_{2} 5 \%$. The bacteria were scraped from the plates with cotton swabs, suspended in phosphate-buffered saline ( $\mathrm{pH} 7 \cdot 2$ ) and heat inactivated for $30 \mathrm{~min}$ at $56^{\circ} \mathrm{C}$. The concentration of the suspensions was adjusted to an optical density (OD) of $c .0 \cdot 100$, measured in a standard $1-\mathrm{cm}$ cuvette at $620 \mathrm{~nm}$ with a Titertek Multiscan (Flow Laboratories). The suspensions were stored at $4^{\circ} \mathrm{C}$ until processed further.

\section{Serotyping and subtyping}

Serotyping and subtyping were performed by a WCE, as described previously. ${ }^{23}$ The panel of MAbs included antibodies against serotypes 1, 2a, 2b, 4, 14, 15 and 16, and subtypes P1.1, P1.2, P1.4, P1.5, P1.6, P1.7, P1.9, P1.10, P1.12, P1.14, P1.15 and P1.16. The MAb against subtype P1.5 (Mn22A9.19) has been developed recently and was shown to be specific for the class 1 outer-membrane protein (OMP) by immunoblotting analysis.

\section{LOS immunotyping}

LOS immunotyping was done by WCE, as described previously, ${ }^{23}$ with MAbs against LOS of $N$. meningitidis. The MAbs were produced and tested for specificity as described elsewhere. ${ }^{5-7,24,25}$ Generally, outer-membrane complexes were used as immunising and detecting antigens to develop OMP-specific MAbs. ${ }^{24,25}$ Several LOS MAbs have been isolated during the development of OMP MAbs. These have been characterised by immunoblotting and WCE, and the most useful ones were used in this study. MAbs Mn4D1B3 and Mn4A8B2 were made after immunisation with oligosaccharide-tetanus toxoid conjugates. ${ }^{26}$ The panel of MAbs is shown in table I. MAbs 17-1-L1, 9-2-L3,7,9, 2-1-L8 and 14-1-L10 were from the laboratory of W.D.Z. All MAbs are of the IgG class, except Mn4D1B3, which was IgM. For this $\mathrm{MAb}$, anti-mouse immunoglobulin was used as a conjugate in the WCE instead of protein-A-peroxidase. The ELISA results were read with a Titertek Multiscan (Flow Laboratories) at $450 \mathrm{~nm}$. The OD cut-off values for a positive reaction in the WCE depended on the MAb, the non-specific background value of negative controls and the intensity of the reaction with positive controls. The reaction pattern of each isolate to the panel was determined on the basis of the individual OD cut-off values of the MAbs.

To assess the comparability of the WCE method with the microprecipitation method for immunotyping, the WCE results of the 57 isolates collected in 1974 were compared with the results obtained previously by microprecipitation. ${ }^{4}$

To assess the reproducibility of the WCE method for immunotyping, the 57 isolates obtained in 1974 , as well as 59 of the isolates obtained in 1989-1990, were cultured and immunotyped twice, and both ITs of each of the 116 pairs were compared.

\section{Results}

\section{Reference strains}

Table I shows the reactions of the 14 MAbs with the 11 LOS reference strains in the WCE. Five MAbs reacted exclusively with one reference strain: Mn14F21-11 and 17-1-L1 with strain 126E (L1), 14-1L10 with 7880 (L10), Mn3A8C with M981 (L5) and Mn4C1B with M992 (L6). The other nine MAbs reacted with two or more reference strains, indicating the presence of shared or additional LOS epitopes on some reference strains.

\section{Assignment of LOS immunotypes}

On the basis of the observed reaction patterns of the 675 patient isolates from $1989-1990$ with the 14 MAbs, and the knowledge of the reactions of the various MAbs with the reference strains, an algorithm for the 
Table I. MAbs used for LOS immunotyping of N. meningitidis and results of immunotyping of 11 LOS reference strains in a WCE

\begin{tabular}{|c|c|c|c|c|c|c|c|c|c|c|c|}
\hline \multirow[b]{2}{*}{$\mathrm{MAb}$} & \multicolumn{11}{|c|}{ OD in WCE with strain no. (immunotype) } \\
\hline & $\begin{array}{l}126 \mathrm{E} \\
\text { (L1) }\end{array}$ & $\begin{array}{l}35 \mathrm{E} \\
(\mathrm{L} 2)\end{array}$ & $\begin{array}{l}6275 \\
\text { (L3) }\end{array}$ & $\begin{array}{c}89 \mathrm{I} \\
(\mathrm{L} 4)\end{array}$ & $\begin{array}{c}\text { M981 } \\
\text { (L5) }\end{array}$ & $\begin{array}{c}\text { M992 } \\
\text { (L6) }\end{array}$ & $\begin{array}{l}6155 \\
(\mathrm{~L} 7)\end{array}$ & $\begin{array}{c}\text { M978 } \\
\text { (L8) }\end{array}$ & $\begin{array}{c}\text { 120M } \\
\text { (L9) }\end{array}$ & $\begin{array}{c}7880 \\
(\mathrm{Ll} 10)\end{array}$ & $\begin{array}{r}7889 \\
(\mathrm{~L} 11)\end{array}$ \\
\hline $\mathrm{Mn} 14 \mathrm{~F} 21-11$ & $1 \cdot 8$ & $0 \cdot 1$ & $0 \cdot 1$ & $0 \cdot 1$ & $0 \cdot 1$ & 0.1 & $0 \cdot 1$ & $0 \cdot 1$ & $0 \cdot 1$ & $0 \cdot 1$ & $0 \cdot 1$ \\
\hline $17-1-\mathrm{Ll}$ & $2 \cdot 4$ & $0 \cdot 2$ & 0.2 & $0 \cdot 2$ & $0 \cdot 2$ & $0 \cdot 2$ & $0 \cdot 2$ & 0.3 & $0 \cdot 3$ & $0 \cdot 1$ & $0 \cdot 2$ \\
\hline Mn14F20-11 & $0 \cdot 6$ & $0 \cdot 1$ & $0 \cdot 1$ & $0 \cdot 1$ & $0 \cdot 1$ & $0 \cdot 1$ & $0 \cdot 2$ & $2 \cdot 5$ & $0 \cdot 2$ & 0.5 & 1.7 \\
\hline $\mathrm{Mn} 13 \mathrm{H} 21$ & $1 \cdot 3$ & 0.2 & 0.4 & 0.1 & $0 \cdot 1$ & $0 \cdot 1$ & 0.6 & $2 \cdot 2$ & $1 \cdot 4$ & 1.8 & 1.9 \\
\hline $2-1-L 8$ & $1 \cdot 7$ & $0 \cdot 3$ & 0.6 & 0.2 & $0 \cdot 2$ & $0 \cdot 2$ & 0.6 & $2 \cdot 4$ & $1 \cdot 1$ & $1 \cdot 4$ & $1 \cdot 7$ \\
\hline 14-1-L10 & $0 \cdot 1$ & $0 \cdot 1$ & $0 \cdot 1$ & $0 \cdot 1$ & $0 \cdot 1$ & $0 \cdot 1$ & 0.1 & $0 \cdot 1$ & $0-1$ & 1.9 & $0 \cdot 1$ \\
\hline $\mathrm{Mn} 11 \mathrm{Al} 1 \mathrm{G}$ & $0 \cdot 1$ & 0.2 & 0.8 & $0 \cdot 1$ & $0 \cdot 1$ & $0 \cdot 1$ & 0.1 & $0 \cdot 1$ & 0.3 & 0.2 & $0 \cdot 6$ \\
\hline $\mathrm{Mn} 15 \mathrm{~A} 17 \mathrm{~F} 12$ & $0 \cdot 1$ & 0.8 & $1 \cdot 7$ & 0.3 & 0.8 & $0 \cdot 1$ & 1.8 & 0.6 & $2 \cdot 1$ & $2 \cdot 0$ & $0 \cdot 1$ \\
\hline 9-2-L3,7,9 & $0 \cdot 1$ & $1 \cdot 0$ & $2 \cdot 3$ & $0 \cdot 3$ & 0.7 & $0 \cdot 1$ & $2 \cdot 4$ & $2 \cdot 2$ & $2 \cdot 4$ & $0 \cdot 2$ & $0 \cdot 1$ \\
\hline Mn15A8-1 & $0 \cdot 2$ & $2 \cdot 0$ & $2 \cdot 3$ & $1 \cdot 7$ & $2 \cdot 1$ & 0.2 & $2 \cdot 1$ & 1.9 & $2 \cdot 3$ & 0.2 & $0 \cdot 1$ \\
\hline $\mathrm{Mn} 4 \mathrm{D} 1 \mathrm{~B} 3$ & $0 \cdot 2$ & 0.5 & $1 \cdot 6$ & $0 \cdot 2$ & $0 \cdot 2$ & $0 \cdot 2$ & $1 \cdot 7$ & $1 \cdot 3$ & $1 \cdot 6$ & $0 \cdot 2$ & 0.2 \\
\hline Mn4A8B2 & $0 \cdot 1$ & $0 \cdot 1$ & $1 \cdot 5$ & $0 \cdot 1$ & $0 \cdot 1$ & $0 \cdot 1$ & $1 \cdot 6$ & $0 \cdot 3$ & $1 \cdot 6$ & 0.1 & $0 \cdot 1$ \\
\hline $\mathrm{Mn} 3 \mathrm{~A} 8 \mathrm{C}$ & $0 \cdot 1$ & $0 \cdot 1$ & $0 \cdot 1$ & $0 \cdot 1$ & 1.9 & $0 \cdot 1$ & $0 \cdot 1$ & $0 \cdot 1$ & $0 \cdot 1$ & $0 \cdot 1$ & $0 \cdot 1$ \\
\hline $\mathrm{Mn} 4 \mathrm{ClB}$ & $0 \cdot 1$ & $0 \cdot 2$ & $0 \cdot 1$ & 0.3 & 0.1 & $1 \cdot 4$ & $0 \cdot 1$ & 0.1 & 0.4 & $0 \cdot 1$ & 0.1 \\
\hline
\end{tabular}

Table II. Algorithm for the assignment of LOS ITs of $N$. meningitidis as determined in a WCE with a panel of 14 MAbs

\begin{tabular}{|c|c|c|c|c|c|c|c|c|c|}
\hline \multirow{2}{*}{$\mathrm{MAb}$} & \multicolumn{9}{|c|}{ Reaction given by immunotype } \\
\hline & L1 & $\mathbf{L} 2 \dagger$ & L3 & L4 & L5 & L6 & L8: & L10 & L11 \\
\hline Mn14F21-11 and/or 17-1-L1 & + & & & & & & & & \\
\hline Mn14F20-11 & & - & & - & & & + & & \\
\hline $\mathrm{Mn} 13 \mathrm{H} 21$ and/or 2-1-L8 & & - & & - & & & & & \\
\hline 14-1-L10 & & & & & & & & + & \\
\hline $\mathrm{Mn} 11 \mathrm{~A} 11 \mathrm{G}$ & & - & & - & & & & & + \\
\hline Mn15A17F12 & & + & & - & & & & & \\
\hline $9-2-\mathrm{L} 3,7,9$ & & + & & - & & & & & \\
\hline Mn15A8-1 & & + & & + & & & & & \\
\hline $\mathrm{Mn} 4 \mathrm{D} 1 \mathrm{~B} 3$ & & - & & - & & & & & \\
\hline $\mathrm{Mn} 4 \mathrm{~A} 8 \mathrm{~B} 2$ & & - & + & - & & & & & - \\
\hline $\mathrm{Mn} 3 \mathrm{~A} 8 \mathrm{C}$ & & - & & - & + & & & & \\
\hline $\mathrm{Mn} 4 \mathrm{C} 1 \mathrm{~B}$ & & & & & & + & & & \\
\hline
\end{tabular}

+ , positive reaction; - , negative reaction.

$\div \mathrm{L} 2$ was assigned in the case of a positive reaction with Mn15A8-1 and either or both of MAbs Mn15A17F12 and 9-2-L3,7,9.

$\$$ L11 was assigned in the case of a positive reaction with both Mn14F20-11 and Mn11A11G and a negative reaction with Mn4A8B2.

Table III. Examples of reaction patterns of isolates of various ITs and IT combinations to a panel of 14 MAbs in a WCE

\begin{tabular}{|c|c|c|c|c|c|c|c|c|c|c|c|c|c|c|c|c|c|c|c|c|}
\hline \multirow[b]{2}{*}{$\mathrm{MAb}$} & \multicolumn{20}{|c|}{ Reaction obtained with strain no. and IT } \\
\hline & $\begin{array}{c}892633 \\
\text { L1 }\end{array}$ & $\begin{array}{c}900997 \\
\text { L1,8 }\end{array}$ & $\begin{array}{c}901005 \\
\text { L1 }, 8\end{array}$ & $\begin{array}{c}891357 \\
\text { L2 }\end{array}$ & $\begin{array}{c}892385 \\
\text { L3 }\end{array}$ & $\begin{array}{c}900755 \\
\text { L3 }\end{array}$ & $\begin{array}{c}900820 \\
\text { L3 }\end{array}$ & $\begin{array}{c}892463 \\
\text { L3,1 }\end{array}$ & $\begin{array}{c}891177 \\
\text { L3,1 }\end{array}$ & $\begin{array}{c}900747 \\
\mathrm{~L} 3,1,8\end{array}$ & $\begin{array}{c}891021 \\
\text { L3,8 }\end{array}$ & $\begin{array}{c}892214 \\
\text { L4 }\end{array}$ & $\begin{array}{c}\text { M981 } \\
\text { L5 }\end{array}$ & $\begin{array}{c}\text { M992 } \\
\text { L6 }\end{array}$ & $\begin{array}{c}900714 \\
\text { L8 }\end{array}$ & $\begin{array}{c}891165 \\
\text { L8 }\end{array}$ & $\begin{array}{c}900018 \\
\text { L8,10 }\end{array}$ & $\begin{array}{c}900100 \\
\text { L10 }\end{array}$ & $\begin{array}{l}892411 \\
L 10,11\end{array}$ & $\begin{array}{c}7889 \\
\text { L11 }\end{array}$ \\
\hline Mn14F21-11/17-1-L1 & + & + & + & & & & & + & + & + & & & & & & & & & & \\
\hline $\mathrm{Mn} 14 \mathrm{~F} 20-11$ & $\mathrm{w}$ & + & + & & & & & & & + & + & & & & + & + & + & & + & + \\
\hline $\mathrm{Mn} 13 \mathrm{H} 2 \mathrm{l} / 2-\mathrm{I}-\mathrm{L} 8$ & + & + & + & & & w & + & & w & + & + & & & & + & + & + & $w$ & + & + \\
\hline 14-1-L10 & & & & & & & & & & & & & & & & & + & + & + & \\
\hline MnllAllG & & & & & + & & + & & & & & & & & & & & $w$ & + & + \\
\hline Mn15A17F12 & & & w & + & + & + & + & + & + & + & + & & & & & + & + & + & + & \\
\hline $9-2-\mathrm{L} 3,7,9$ & & & + & + & + & + & + & + & + & + & + & & w & & w & + & & & & \\
\hline Mn15A8-1 & & & + & + & + & + & + & + & + & + & + & + & + & & w & + & & & & \\
\hline $\mathrm{Mn} 4 \mathrm{D} 1 \mathrm{~B} 3$ & & & & & + & + & + & + & + & + & + & & & & & w & & & & \\
\hline Mn4A8B2 & & & & & + & + & + & + & + & + & + & & & & & w & & & & \\
\hline $\mathrm{Mn} 3 \mathrm{~A} 8 \mathrm{C}$ & & & & & & & & & & & & & + & & & & & & & \\
\hline $\mathrm{Mn} 4 \mathrm{ClB}$ & & & & & & & & & & & & & & + & & & & & & \\
\hline
\end{tabular}

+ , Positive reaction; $w$, weak positive reaction.

assignment of ITs was developed (table II). The following ITs were discerned: L1, L2, L3, L4, L5, L6, L8, L10 and L11. L7 and L9 could not be separated from L3 by this set of MAbs, and were considered to be L3. An isolate was labelled L3 if it reacted with MAb Mn4A8B2. Almost all L3 isolates also reacted with MAbs Mn4D1B3, Mn15A17F12, 9-2-L3,7,9 and Mn15A8-1, and many with Mn11A11G. An isolate was assigned to L8 if it reacted with Mn14F20-11, with one exception: if an isolate reacted with both Mn14F20-11 and Mn11A11G, and not with Mn4A8B2, it was labelled L11. The majority of the L8 
Table IV. Comparison of LOS ITs of 57 meningococcal isolates obtained by microprecipitation with polyclonal antibodies $^{4}$ and WCE with MAbs

\begin{tabular}{llr}
\hline \multicolumn{2}{c}{ IT determined by } & \\
\cline { 1 - 2 } Microprecipitation & WCE & Number of isolates \\
\hline L1 & L1 & \\
L1 & L1,8 & 1 \\
L1,8 & L1,8 & 1 \\
L1,8 & L8 & 3 \\
L2 & L2 & 2 \\
L2 & L2,1 & 16 \\
L2 & L8 & 1 \\
L2,11 & L2 & 1 \\
L3 & L3 & 1 \\
L3 & L3,8 & 19 \\
L3 & L8 & 4 \\
L3,1,8 & L3,1,8 & 1 \\
L3,1,8 & L3,8 & 2 \\
L6 & L6 & 1 \\
L10 & L10 & 1 \\
Non-typable & L2 & 1 \\
Non-typable & Non-typable & 1 \\
& & 1 \\
\hline
\end{tabular}

Table V. Comparison of LOS ITs of 116 meningococcal isolates of two separate cultures obtained by WCE

\begin{tabular}{llr}
\hline \multicolumn{2}{c}{ IT determined in } \\
\cline { 1 - 1 } \multicolumn{1}{c}{ culture 1 } & \multicolumn{1}{c}{ culture 2 } & \\
\hline L1 & L1 & \\
L1,8 & L1,8 & 2 \\
L2 & L2 & 15 \\
L2 & Non-interpretable & 28 \\
L2,1 & L2 & 2 \\
L3 & L3 & 1 \\
L3 & L3,6 & 30 \\
L3,1 & L3,1 & 1 \\
L3,1 & L3,1,8 & 2 \\
L3,1,8 & L3,1,8 & 1 \\
L3,1,8 & L3,8 & 4 \\
L3,8 & L3 & 1 \\
L3,8 & L3,8 & 2 \\
L4 & L2 & 9 \\
L6 & L6 & 1 \\
L8 & L8 & 1 \\
L8 & L1,8 & 9 \\
L8 & Non-typable & 1 \\
L8,10 & L8,10 & 1 \\
L10 & L10 & 1 \\
Non-typable & Non-typable & 1 \\
Non-interpretable & L2 & 1 \\
Non-interpretable & Non-interpretable & 1 \\
& & $1 *$ \\
& &
\end{tabular}

* Both cultures showed identical MAb reaction patterns.

isolates also reacted with MAbs 2-1-L8 and $\mathrm{Mn} 13 \mathrm{H} 21$. Because there were no immunotype-specific MAbs for $\mathrm{L} 2$ and $\mathrm{L} 4$, the assignment of these immunotypes was based on the reaction pattern of an isolate with several MAbs. Isolates were assigned to L2 if a positive reaction was found with Mn15A8-1 in combination with either one or both of MAbs Mn15A17F12 and 92-L3,7,9, and negative reactions with MAbs Mn4A8B2, Mn4D1B3, Mn11A11G, Mn3A8C, $\mathrm{Mn} 13 \mathrm{H} 21,2-1-\mathrm{L} 8$ and $\mathrm{Mn} 14 \mathrm{~F} 20-11$. If the latter reaction pattern was observed in combination with negative reactions with both $\mathrm{Mn} 15 \mathrm{~A} 17 \mathrm{~F} 12$ and 9-2L3,7,9, the IT was assumed to be L4. Because the combinations $\mathrm{L} 2,8$ and $\mathrm{L} 4,8$ are assumed not to exist (see Discussion), an additional positive reaction with MAb Mn14F20-11 excluded ITs L2 or L4 and the isolate was assigned to L8. The assignment of isolates to immunotypes L1, L5, L6 and L10 was based on a positive reaction with the specific MAbs described above. All L10 isolates also reacted with Mn15A17F12. Combinations of immunotypes, such as $\mathrm{L} 1,8, \mathrm{~L} 3,1, \mathrm{~L} 3,1,8$, and $\mathrm{L} 3,8$ were also discerned. A positive reaction with $\mathrm{Mn} 15 \mathrm{~A} 8 \mathrm{-1}$ (with or without Mn15A17F12 or 9-2-L3,7,9) on the one hand, and Mn13H21, 2-1-L8 or Mn4D1B3 on the other, was considered non-interpretable in the absence of a positive reaction with one of the other MAbs. Isolates that failed to react with any MAb were labelled nontypable (NT).

\section{ITs and MAb reaction patterns}

Table III shows examples of the reaction patterns of isolates of the various ITs and IT combinations. For ITs L2-L6, isolates with a "pure" reaction pattern were found. However, all L1 and L10 and many L3 strains showed some reaction with the L8 groups of MAbs (Mn13H21 and 2-1-L8), and many L8 isolates with the L3 group (Mn4D1B3, Mn15A17F12, 9-2$\mathrm{L} 3,7,9, \mathrm{Mn} 15 \mathrm{~A} 8-1$ ), indicating that the isolates under consideration partly expressed other ITs. The reference strains 126E (L1), 6275 (L3), M978 (L8) and 7880 (L10) did not show "pure" patterns (table I). It was impossible to assign an IT to 12 of the 675 isolates, because of a non-interpretable reaction pattern. Only five isolates were NT. ITs L5 and L6 were found only among the reference strains.

\section{Comparison of immunotyping by WCE and microprecipitation}

The ITs obtained in the WCE, with MAbs agreed well with those previously obtained by microprecipitation with polyclonal antibodies (table IV). ${ }^{4}$ Of the 57 isolates tested, $44(77 \%)$ showed identical ITs in both methods. For three isolates $(5 \%)$ the results of the two methods disagreed completely. One isolate was immunotypable as L 2 by WCE and yet NT by microprecipitation. A second culture of this isolate was again found to be L2 by WCE. One isolate, which was $\mathrm{L} 2$ by microprecipitation, was repeatedly $\mathrm{L} 8$ by WCE. The third isolate, which was L3 by microprecipitation, was first designated L8 by WCE, whereas a second culture of this isolate was NT by WCE, although it reacted weakly with the L8 group of MAbs. For 10 isolates (18\%) minor differences were observed. Two isolates that were L8 and one that was $\mathrm{L} 3,8$ by WCE were typed $\mathrm{L} 1,8$ and $\mathrm{L} 3,1,8$, respectively, by microprecipitation. However, it was not possible to discriminate between $\mathrm{L} 8$ and $\mathrm{L} 1,8$ or $\mathrm{L} 3,8$ and $\mathrm{L} 3,1,8$ by microprecipitation with polyclonal antibodies. 
Table VI. Distribution of LOS ITs/serogroup among 562 meningococcal isolates from patients with meningococcal disease in the Netherlands, 1989-1990

\begin{tabular}{llcccc}
\hline & \multicolumn{5}{c}{ Number (\%) } \\
& IT & \multicolumn{1}{c}{ strains of serogroup } \\
\cline { 2 - 6 } & & B & C & Other & Total \\
\hline L1 & 0 & $3(1)$ & 0 & 0 & $3(1)$ \\
L1,8 & 0 & $46(11)$ & $2(2)$ & $1(5)$ & $49(9)$ \\
L2 & 0 & $42(10)$ & $31(30)$ & $7(37)$ & $80(14)$ \\
L2,1 & 0 & $1(0)$ & 0 & 0 & $1(0)$ \\
L3 & $7(54)$ & $151(36)$ & $39(37)$ & $7(37)$ & $204(37)$ \\
L3,1 & 0 & $16(4)$ & $1(1)$ & $1(5)$ & $18(3)$ \\
L3,1,8 & 0 & $7(2)$ & $3(3)$ & 0 & $10(2)$ \\
L3,8 & $1(8)$ & $115(28)$ & $7(7)$ & 0 & $123(22)$ \\
L4 & 0 & $16(4)$ & $16(15)$ & $2(11)$ & $34(6)$ \\
L8 & 0 & $19(5)$ & $3(3)$ & 0 & $22(4)$ \\
L8,10 & 0 & $2(0)$ & 0 & 0 & $2(0)$ \\
L10* & $4(31)$ & 0 & 0 & 0 & $4(1)$ \\
Non-typable & $1(8)$ & 0 & $3(3)$ & $1(5)$ & $5(1)$ \\
Non-interpretable & 0 & 7 & 0 & 0 & 7 \\
Total & 13 & 425 & 105 & 19 & 562 \\
& & & & & \\
\hline
\end{tabular}

* Includes one $\mathrm{L} 10,11$ isolate.

\section{Reproducibility of the WCE method for LOS- immunotyping}

The results of immunotyping by WCE of two separate cultures of 116 isolates are shown in table $\mathrm{V}$. Both ITs were identical for 104 pairs $(90 \%)$; seven pairs $(6 \%)$ showed minor differences, and in five pairs $(4 \%)$ a major difference was found. The major difference for one pair (L8 and NT, respectively) pertained to an isolate that also showed a different result when WCE was compared with microprecipitation. The second culture of this isolate reacted weakly with Mn14F20-11 (L8). In three discordant pairs, one of the isolates was typed as L2 and the other showed a non-interpretable result. In the last discordant pair, the first isolate was L4 and the second L2. In five of the seven pairs with a minor difference, the difference was due to a weak reaction with a MAb essential for that specific IT assignment, and in two instances there seemed to be a true additional IT in one of the two cultures from each isolate.

\section{Distribution of LOS ITs among meningococcal isolates obtained in 1989-1990}

Isolates from both CSF and blood were obtained from 112 of the 563 patients. In the majority of the 112 paired strains, both ITs were identical. Only 16 pairs $(14 \%)$ showed a difference. In five of these 16 pairs the differences were striking. One pair showed totally discordant IT results (L2 and $\mathrm{L} 1,8$ ) and these two isolates did not have a positive reaction in common with any of the 14 MAbs. In one pair, the blood isolate was L2 and the CSF isolate L4. In three instances, one isolate of each pair reacted strongly with Mn4A8B2 (L3), whereas the other did not react (two isolates), or reacted only weakly (one isolate). In the 11 pairs with only minor differences, the differences pertained to the presence or absence of an additional L8, but in 10 pairs the non-L8 isolate reacted weakly with Mn14F20-11 (L8), indicating the presence, although weakly expressed, of an additional L8 IT. In the analyses of the results, only one strain from each patient was included. The L2-L4 pair was considered to be L2, and the other 14 different pairs were considered to be L3,8. The completely discordant pair was omitted, leaving 562 isolates for further analyses.

Table VI shows the distribution of LOS ITs by serogroup among 562 meningococci obtained in 1989-1990 from patients with meningococcal disease. The most prevalent ITs are L3 $(37 \%), \mathrm{L} 3,8(22 \%)$, L2 $(14 \%), \mathrm{L} 1,8(9 \%)$ and $\mathrm{L} 4(6 \%)$. The distribution of ITs differed in the various serogroups. The 13 serogroup A isolates were either $\mathrm{L} 10$ (one isolate expressed both $\mathrm{L} 10$ and L11), or L3 or a related IT, and one isolate was NT. In 425 serogroup B isolates, seven showed a non-interpretable IT result. L3 and the related ITs $\mathrm{L} 3,1, \mathrm{~L} 3,1,8$ and $\mathrm{L} 3,8$ predominated $(69 \%)$, and L2 and L4 accounted for $10 \%$ and $4 \%$, respectively. In contrast, almost half of the 105 serogroup C isolates were L2 or L4 $(30 \%$ and $15 \%$, respectively). L1, L8 and $\mathrm{L} 1,8$ comprised $17 \%$ of the serogroup B and $5 \%$ of the serogroup $\mathrm{C}$ isolates. Other serogroups, which were rare, gave various ITs.

\section{Association of IT and sero(sub)type}

Of the 13 serogroup A isolates, seven were $\mathrm{A}: \mathrm{NT}: \mathrm{P} 1.16: \mathrm{L} 3$. The two $\mathrm{A}: 4: \mathrm{P} 1.10$ isolates were $\mathrm{L} 10$ and $\mathrm{L} 10,11$ respectively, and of the three $\mathrm{A}: 4: \mathrm{P} 1.9$ isolates, two were L10 and one was NT. The exotic $\mathrm{A}: 15: \mathrm{NT}$ isolate was $\mathrm{L} 3,8$.

Within both serogroups B and C, L2 and L4 were strongly associated with the serotypes $2 \mathrm{a}$ and $2 \mathrm{~b}$. Of the $15 \mathrm{~B}: 2 \mathrm{a}$ isolates, three $(20 \%)$ were $\mathrm{L} 2$ and 10 $(67 \%)$ were $\mathrm{L} 4$, and of the $44 \mathrm{C}: 2 \mathrm{a}$ isolates, $18(41 \%)$ were L2 and $10(23 \%)$ were L4. L2 and L4 were found in $45 \%$ and $9 \%$ of the $11 \mathrm{~B}: 2 \mathrm{~b}$ isolates, and in $31 \%$ and $8 \%$ of the $13 \mathrm{C}: 2 \mathrm{~b}$ isolates, respectively. Among the serotype $2 \mathrm{a}$ and $2 \mathrm{~b}$ isolates of these serogroups, no L1, L1,8 or L8 was found. L3 and related ITs were present in $124(69 \%)$ of $181 \mathrm{~B}: 4$ isolates and in 38 $(88 \%)$ of the $43 \mathrm{~B}: 15$ isolates $(88 \%)$, whereas the percentages of the L1/L8 category of ITs were $25 \%$ and $7 \%$, respectively. Serogroup $\mathrm{B}$ isolates with subtype $\mathrm{P} 1.4, \mathrm{P} 1.5, \mathrm{P} 1.15, \mathrm{P} 1.16$ or $\mathrm{P} 1.7,16$ were mainly of the L3 and L1/L8 category of ITs, ranging from 71 to $91 \%$ and 0 to $26 \%$, respectively, but those with the subtypes P1.2 or P1.5,2 were mainly associated with $\mathrm{L} 2(57 \%$ and $15 \%)$ and $\mathrm{L} 4(14 \%$ and $30 \%)$. The latter association also applied for serogroup C: all three P1.2 isolates were $\mathrm{L} 2$, and of the $36 \mathrm{P} 1.5,2$ isolates, $17(47 \%)$ were L2 and eight $(22 \%)$ were L4. Of the 16 subtype $\mathrm{P} 1.5$ isolates of serogroup C, L3 and related ITs were found in $69 \%$. Among the 116 isolates of the most prevalent phenotype (B:4:P1.4) in the Netherlands, only three ( $3 \%$ ) were L2, $83(72 \%)$ were L3 or a related IT, and $30(26 \%)$ were of the L1/L8 category. For the 31 isolates of the "Norwegian" 
phenotype B:15:P1.(7,)16, these percentages were $6 \%, 94 \%$ and $0 \%$, respectively.

The other serogroups were heterogeneous with regard to both sero(sub)type and IT.

\section{Discussion}

The various surface structures of $N$. meningitidis are useful tools for studying the spread of meningococcal disease. In the past decade several MAbs against LOS have been developed. This has enabled immunotyping by WCE to be performed easily and on a large scale. Despite this improvement, only a few studies concerning $N$. meningitidis have included LOS ITs. A reason for this might be the absence of specific MAbs for some ITs, such as L2 and L4. In this study, MAbs Mn15A8-1, Mn15A17F12 and 9-2-L3,7,9 were found to react with the $\mathrm{L} 2$ reference strain and Mn15A8-1 with the L4 reference strain. These MAbs, in combination with 11 others, enabled an algorithm to be developed whereby the ITs are assigned on the basis of the reaction pattern of the isolates to this panel of MAbs. ITs L1, L5, L6 and L10 are easily recognisable, because specific MAbs are available. MAbs Mn4A8B2 and Mn14F20-11 were found to be suitable for determining L3 and L8, respectively. L3, L7 and L9, which are immunochemically identical,${ }^{3,4}$ could not be separated from each other by the panel of MAbs. Because the chemical structures of L 7 and L9 are not known, isolates of this IT complex are labelled L3. ITs $\mathrm{L} 2$ and $\mathrm{L} 4$ are assigned by exclusion (based on negative reactions with other MAbs, apart from a positive reaction with Mn15A8-1 and either one or both of Mn15A17F12 and 9-2-L3,7,9). Therefore, inaccuracy in the laboratory can lead to errors and misclassification. However, the immunotyping results obtained by WCE with MAbs, and this algorithm were similar to those obtained in an earlier study by microprecipitation with polyclonal antibodies. ${ }^{4}$ A discordant IT result was obtained with only three of 57 strains. Because of the lack of a gold standard, these differences could be due to errors in either of the two methods. However, for two of the strains the difference may be attributed to the microprecipitation method, because the ITs of two separate cultures of the same isolate determined by WCE were identical. In 10 strains, subtle differences occurred, indicating the presence of additional ITs among related ITs (see below). Unfortunately, no L4 strain was present among these 57 isolates tested for comparability. Therefore, the exact assignment of L4, remains uncertain.

The immunotyping results obtained by the use of the algorithm were reproducible. When comparing the ITs determined by WCE of two separate cultures of the same isolate, only five $(4 \%)$ discordant pairs among 116 pairs were found. In three of these pairs, one of the cultures resulted in L2 and the other in a non-interpretable result. The difference in the fourth pair (L4-L2) was inherent in the method used, and in the last pair a shift from L8 to NT was found, possibly due to laboratory error. This also seems to have been the cause of five of the seven minor differences between the paired test results. The remaining two minor differences could have been caused by plate effects, which seem to occur but only rarely.

The algorithm is appropriate for LOS immunotyping of serogroup $\mathrm{B}$ and $\mathrm{C}$ meningococci. For serogroup $\mathrm{A}$, the scheme is less suitable, as no $\mathrm{MAb}$ is yet available for $\mathrm{L} 11$ or the recently discovered ITs L12 and L13. ${ }^{19}$ The assignment of isolates to L11 is possible, but rather complicated. However, there were remarkable findings among the 13 serogroup A meningococci in our study. Of the five group $A$ isolates with a typical group A serotype-subtype combination (4:P1.9 and 4:P1.10), four isolates expressed the L10 IT and one was NT. The eight remaining serogroup A isolates were of a serotype (serotype 15) or subtype (P1.16), which are typical of serogroup B, as are L3 and L3,8, which were also expressed by these isolates. This suggests a recent switch of the capsule of strains that were originally serogroup B to serogroup A.

Based on the reaction patterns found among serogroup $\mathrm{B}$ and $\mathrm{C}$ meningococci, two main categories of related ITs can be found within these serogroups; one category is $\mathrm{L} 2 / \mathrm{L} 4$, and the other $\mathrm{L} 3 / \mathrm{L} 1 / \mathrm{L} 8$. In view of the chemical structure of the different ITs (figure), it is conceivable that an $\mathrm{L} 3$ strain is able to express also $\mathrm{L} 1$ or L8, or both, because the terminal structures of these three ITs differ only slightly. ${ }^{13-16,18}$ Considering the reaction patterns of isolates belonging to the L3/L1/L8 category, all L1 and many L3 strains reacted weakly with the $\mathrm{L} 8$ group of MAbs, and many L8 strains reacted weakly with the L3 group. Therefore, the differences in this category seem to be of a quantitative rather than a qualitative nature. This suggests the existence of a related group of $\mathrm{L} 3, \mathrm{Ll}$ and L8 ITs with subtle or more pronounced IT varieties and a possible shift of ITs. In this respect, it is remarkable that in Britain a major difference has been discovered between carrier isolates (mostly L1/L8) and case isolates $(\mathrm{L} 3)^{27}$

The reference strains of the L3/L1/L8 group of ITs did not show "pure" reaction patterns. Strain $126 \mathrm{E}$ (L1) also reacted with the L8 group of MAbs, strain M978 (L8) with the L3 group and strain 6275 (L3) weakly with the L8 group (table I). None of our isolates showed a "pure" $\mathrm{Ll}$ reaction pattern, but strain no. 892385 (table III) reacted solely with the L3 group. Strain no. 900714 (table III) seemed to perform better than the original L8 reference strain M978 (table I), although it reacted weakly with two of the L3 group of MAbs. The same reasoning may apply to strain no. 900100 as a reference strain for L10. The above-mentioned strains from our series could be used as new reference strains until new strains expressing a single IT have been constructed by recombinant DNA technology.

With the chemical structure in mind, the dissimilarity between the $\mathrm{L} 2 / \mathrm{L} 4$ and $\mathrm{L} 3 / \mathrm{L1} / \mathrm{L} 8$ categories is 

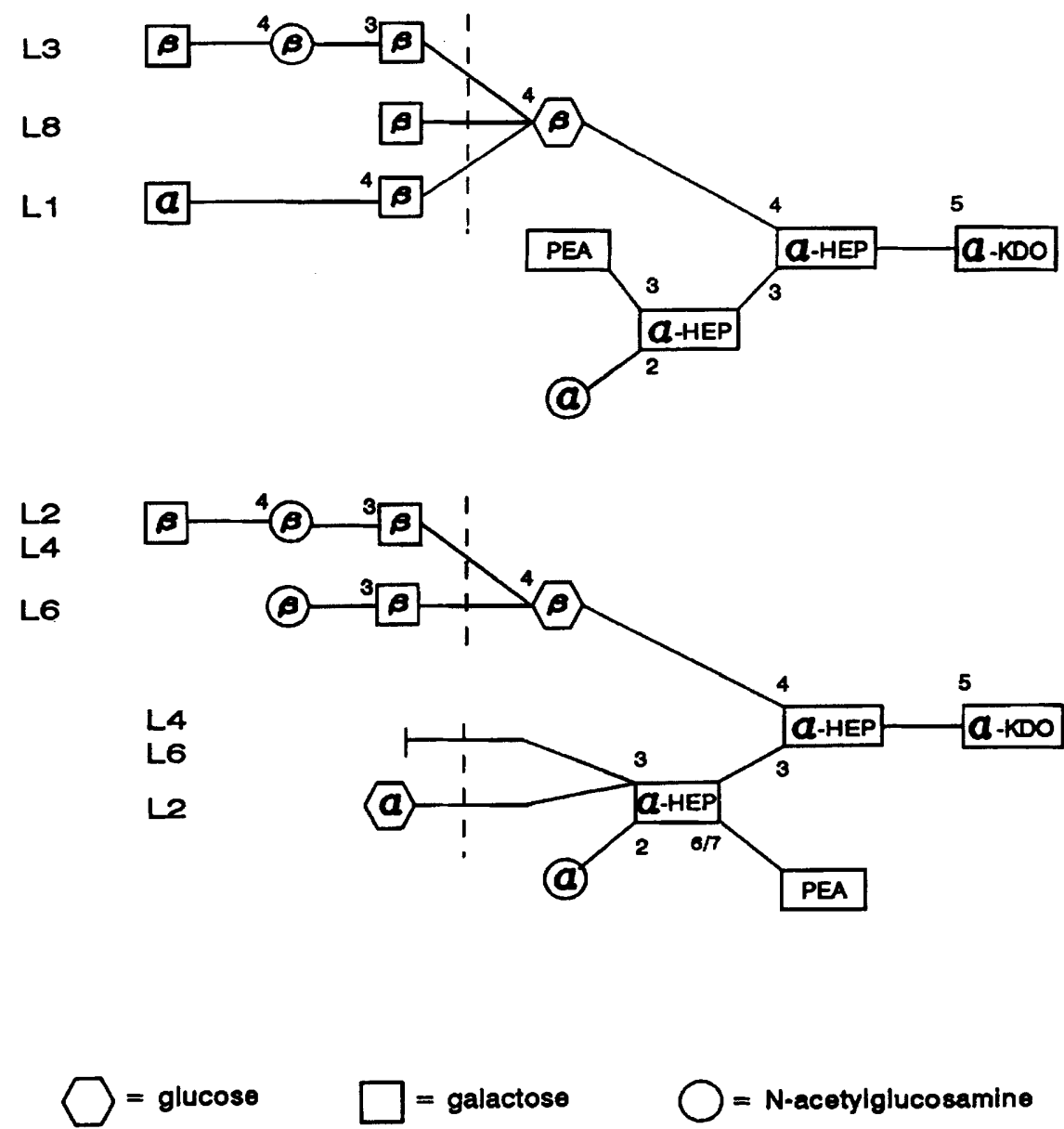

$O=\mathrm{N}$-acetylglucosamine

Figure. Primary structure of meningococcal oligosaccharides of ITs L1, L2, L3, L4, L6 and L8. ${ }^{13-16,18}$ Differences between the various immunotypes are presented on the left of the dotted line. The arabic numbers indicate the position of the binding site; $\alpha, \beta$ indicate the anomeric configuration. KDO, 2-keto-3-deoxyoctulosonic acid; PEA, phosphoethanolamine; HEP, heptose.

based on the location of the phosphoethanolamine group, and a shift of ITs between those two categories is very unlikely. We have not found ITs of these unrelated categories in the same pair of any of the 116 isolates which were cultured twice. Among the 112 pairs of isolates, obtained from the blood and CSF of the same patient, one pair showed unrelated ITs (L2 and $\mathbf{L} 1,8)$. An erroneous interchange of isolates of two different patients could have taken place, because the reaction patterns of the two isolates differed completely. The occurrence of L1 among L2 or L4 isolates is theoretically possible. We have found this combination only rarely, and doubt its true existence.

During 1989-1990, L2, L3 and L4 were the most prevalent ITs among serogroup $B$ and $C$ meningococci in the Netherlands. Among these serogroups, two main categories of related serotypes, subtypes and immunotypes could be distinguished during this period. The serotypes 4 and 15, the subtypes P1.4, $\mathrm{P} 1.5, \mathrm{P} 1.15, \mathrm{P} 1.16$ and $\mathrm{P} 1.7,16$ were associated with the ITs of the L3/L1/L8 category, and the serotypes $2 \mathrm{a}$ and $2 \mathrm{~b}$, the subtypes P1.2 and P1.5,2 with the ITs L2 and L4. The first category was typical of serogroup $\mathrm{B}$, and the second of serogroup C. The B:4:P1.4 phenotype, which is at present the most prevalent in the Netherlands, ${ }^{28}$ belonged almost exclusively to the L3 complex, as did the "Norwegian" B: 15:P1.7,16 phenotype in the Netherlands. ${ }^{29}$ The epidemiological value of LOS immunotyping seems to be limited compared to the other meningococcal surface markers, because there are only a few different IT categories and the IT differences within the $\mathrm{L} 3 / \mathrm{L} 1 / \mathrm{L} 8$ category apparently are more quantitative than qualitative.

The LOS IT is considered to be a virulence determinant. ${ }^{27}$ Isolates of ITs with a terminal lacto-N neotetraose unit (L2-L5, L7 and L9) are capable of endogenously sialylating their LOS, which renders them seroresistant by down-regulation of the alternative complement pathway. ${ }^{10,11}$ L1, L6 and L8 strains are not structured in this way, and are serosensitive. ${ }^{10,11}$ Theoretically, serosensitive strains should be isolated more often from carriers, and this indeed was found to be the case in Britain. ${ }^{27}$ However, a relatively large proportion $(13 \%)$ of $\mathrm{Ll}, \mathrm{L} 1,8$ and $\mathrm{L} 8$ strains, not expressing or only weakly expressing L3, was found among our patient isolates in the period 1989-1990. It is possible that a carrier strain of the L1/L8 category shifts to the L3 group during colonisation, enabling the isolate to evade the host defence mechanisms and cause disease. The high proportion of these $\mathrm{Ll} / \mathrm{L} 8$ isolates among our patient strains could be due to a shift from L3 to L1/L8. A similar association could be postulated for L2, L4 and L6, but we did not find any L6 isolates or combination 
of L6 with L2 or L4 amongst our patient isolates from this period. Unfortunately, no carrier strains were available for this study.

This study has demonstrated that it is possible to carry out meaningful LOS immunotyping of meningococci by the use of carefully selected MAbs, despite the lack of specific MAbs for L2, L4 and L11. The assignment of these ITs is complicated and the development of specific MAbs is warranted. The method is easily applicable for epidemiological research, and provides the possibility of better definition of test strains for in-vitro bactericidal assays and pathogenesis studies. Future studies to determine the

\section{References}

1. Frasch CE, Zollinger WD, Poolman JT. Serotype antigens of Neisseria meningitidis and a proposed scheme for designation of serotypes. Rev Infect Dis 1985; 7: 504-510.

2. Tsai C-M, Mocca LF, Frasch CE. Immunotype epitopes of Neisseria meningitidis lipopolysaccharide types 1 through 8. Infect Immun 1987; 55: 1652-1656.

3. Zollinger WD, Mandrell RE. Outer-membrane protein and lipopolysaccharide serotyping of Neisseria meningitidis by inhibition of a solid phase radioimmunoassay. Infect Immun 1977; 18: 424-433.

4. Poolman JT, Hopman CTP, Zanen HC. Problems in the definition of meningococcal serotypes. FEMS Microbiol Lett 1982; 13: 339-348.

5. Kim JJ, Mandrell RE, Zhen H, Westerink MAJ, Poolman JT, Griffiss JM. Electromorphic characterization and description of conserved epitopes of the lipooligosaccharides of group A Neisseria meningitidis. Infect Immun 1988; 56: 2631-2638.

6. Crowe BA, Wall RA, Kusecek B et al. Clonal and variable properties of Neisseria meningitidis isolated from cases and carriers during and after an epidemic in the Gambia, West Africa. $J$ Infect Dis 1989; 159: 686-700.

7. Saukkonen K, Leinonen M, Abdillahi H, Poolman JT. Comparative evaluation of potential components for group B meningococcal vaccine by passive protection in the infant rat and in vitro bactericidal assay. Vaccine 1989; 7 : 325-328.

8. Zollinger WD, Mandrell RE. Type-specific antigens of group A Neisseria meningitidis; lipopolysaccharides and heatmodifiable outer membrane proteins. Infect Immun 1980; 28: $451-458$.

9. Poolman JT, Hopman CTP, Zanen HC. Colony variants of Neisseria meningitidis strain 2996 (B:2b:P1.2): Influence of class- 5 outer membrane proteins and lipopolysaccharides. $J$ Med Microbiol 1985; 19: 203-209.

10. Mandrell RE, Kim JJ, John CM et al. Endogenous sialylation of the lipooligosaccharides of Neisseria meningitidis. $J$ Bacteriol 1991; 173: 2823-2832.

11. Tsai C-M, Civin CI. Eight lipooligosaccharides of Neisseria meningitidis react with a monoclonal antibody which binds lacto-N-neotetraose (Gal $\beta 1-4 \mathrm{GlcNac} \beta 1-3 \mathrm{Gal} \beta 1-4 \mathrm{Glc})$. Infect Immun 1991; 59: 3604-3609.

12. Mandrell RE, Zollinger WD. Lipopolysaccharide serotyping of Neisseria meningitidis by hemagglutination inhibition. Infect Immun 1977; 16: 471-475.

13. Jennings HJ, Johnson KG, Kenne L. The structure of an R-type oligosaccharide core obtained from some lipopolysaccharides of Neisseria meningitidis. Carbohydr Res 1983; 121: 233-241.

14. Jennings HJ, Beurret M, Gamian A, Michon F. Structure and immunochemistry of meningococcal lipooligosaccharides. Antonie Van Leeuwenhoek 1987; 53: 519-522.

15. Dell A, Azadi P, Tiller $\mathbf{P}$ et al. Analysis of oligosaccharide epitopes of meningococcal lipopolysaccharides by fastatom-bombardment mass spectrometry. Carbohydr Res 1990; 200: 59-76. genetic organisation of LOS biosynthetic genes and LOS chemical structures are needed to improve LOS epitope characterisation. This will lead to the construction of IT reference strains that express a single IT, the selection of appropriate MAbs and the definition of sugar structures as targets for protective immunity.

The authors wish to thank all medical microbiologists in the Netherlands for submitting bacterial isolates to the Reference Laboratory and are most grateful to Eileene Rouppe van der Voort for her invaluable technical assistance. This work was partially supported by grant 28-1874 from the Praeventie Fonds.

16. Difabio JL, Michon F, Brisson JR, Jennings HJ. Structures of the $\mathbf{L} 1$ and $\mathbf{L} 6$ core oligosaccharide epitopes of Neisseria meningitidis. Can J Chem 1990; 68: 1029-1034.

17. Michon F, Beurrett M, Gamian A, Brisson, JR, Jennings HJ. Structure of the L5 lipopolysaccharide core oligosaccharide of Neisseria meningitidis. $J$ Biol Chem 1990; 256: 7243-7247.

18. Schneider H, Griffiss JM, Boslego JW, Hitchcock PJ, Zahos KM, Apicella MA. Expression of paragloboside-like lipooligosaccharides may be a necessary component of gonococcal pathogenesis in men. $J$ Exp Med 1991; 174: 1601-1605.

19. Achtman M, Kusecek B, Morelli G et al. A comparison of the variable antigens expressed by clone IV-1 and subgroup III of Neisseria meningitidis serogroup A. J Infect Dis 1992; 165: $53-68$

20. Salih MAM, Danielsson D, Bäckman A, Caugant DA, Achtman M, Olcén P. Characterization of epidemic and nonepidemic Neisseria meningitidis serogroup A strains from Sudan and Sweden. J Clin Microbiol 1990; 28: 1711-1719.

21. Poolman JT. Polysaccharides and membrane vaccines. In: Mizrahi A (ed) Bacterial vaccines. Advances in Biotechnological Processes, vol 13. New York, Wiley-Liss. 1990: 57-86

22. Slaterus KW. Serological typing of meningococci by means of micro-precipitation. Antonie Van Leeuwenhoek 1961; 27: 305-315.

23. Abdillahi H, Poolman JT. Whole-cell ELISA for typing Neisseria meningitidis with monoclonal antibodies. FEMS Microbiol Lett 1987; 48: 367-371.

24. Zollinger WD, Mandrell RE. Importance of complement source in bactericidal activity of human antibody and murine monoclonal antibody to meningococcal group B polysaccharide. Infect Immun 1983; 40: 257-264.

25. Abdillahi H, Poolman JT. Neisseria meningitidis group B serosubtyping using monoclonal antibodies in whole-cell ELISA. Microb Pathog 1988; 4: 27-32.

26. Verheul AFM, Braat AK, Leenhouts JM et al. Preparation, characterization, and immunogenicity of meningococcal immunotype $\mathrm{L} 2$ and $\mathrm{L} 3,7,9$ phosphoethanolamine groupcontaining oligosaccharide-protein conjugates. Infect Immun 1991; 59: 843-851.

27. Jones DM, Borrow R, Fox AJ, Gray S, Cartwright KA, Poolman JT. The lipooligosaccharide immunotype as a virulence determinant in Neisseria meningitidis. Microb Pathog 1992; 13: 219-224.

28. Scholten RJPM, Bijlmer HA, Poolman JT et al. Meningococcal disease in the Netherlands, 1958-1990: a steady increase in the incidence since 1982 partially caused by new serotypes and subtypes of Neisseria meningitidis. Clin Infect Dis 1993; 16: $237-246$

29. Poolman JT, Lind I, Jónsdóttir KE, Frøholm LO, Jones DM, Zanen HC. Meningococcal serotypes and serogroup B disease in north-west Europe. Lancet 1986; 2: 555-558. 